HZPP-9902

Feb. 25, 1999

\title{
On the Dominance of Statistical Fluctuation in the Factorial-Moment Study of Chaos in Low Multiplicity Events of High Energy Collisions f $^{1}$
}

\author{
Liu Lianshou Fu Jinghua Wu Yuanfang \\ Institute of Particle Physics, Huazhong Normal University, Wuhan 430079 China \\ Tel: $02787673313 \quad$ FAX: $02787662646 \quad$ email: liuls@iopp.ccnu.edu.cn
}

\begin{abstract}
It is shown using Monte Carlo simulation that for low multiplicity events the single-event factorial moments are saturated by the statistical fluctuations. The diverse of the event-space moments $C_{p, q}$ of single-event moments with the diminishing of phase space scale, called "erraticity", observed in experiment can readily be reproduced by a flat probability distribution with only statistical fluctuations and therefore does not indicate the existence of chaos as suggested. The possibility of studying chaos in high multiplicity events using erraticity analysis is discussed.
\end{abstract}

PACS number: $13.85 \mathrm{Hd}$

Keywords: Multiparticle production, Statistical Fluctuations, Chaos, Erraticity

\footnotetext{
1 This work is supported in part by the National Natural Science Foundation of China (NSFC) under Grant No.19575021.
} 
Since the experimental observation in 1983 of unexpectedly large local fluctuations in a single event of very high multiplicity recorded by the JACEE collaboration [1], the investigation of non-linear phenomena in high energy collisions has extracted much attention [2]. The anomalous scaling of factorial moments [3] averaged over event sample called intermittency (or fractal) has been proposed for this purpose. Such kind of anomalous scaling has recently been observed successfully in various experiments [四] [5].

Beside the moments averaged over event sample, the importance of the fluctuation of single-event moments inside an event sample has also been stressed [6]. It is shown that this kind of fluctuation is related to the chaotic behavior of the system [6]. A quantity $\mu_{q}$ called entropy index has been introduced [7] as an adequate parameter in measuring the chaotic behavior. The positivity of entropy index $\mu_{q}>0$ is proved to be a criterion for chaos [8]. This method has been given the name of "erraticity analysis" 9].

The idea of studying chaos through the fluctuation of single-event moments in the event space, in addition to the conventional study of intermittency (fractality) through the moments averaged over event sample, is enlightening. However, the method of eliminating statistical fluctuations using factorial moments, which worked well for the moments averaged over event sample [3], can not be simply extended to the case of single-event moments, cf. Appendix.

In this letter we will take this problem into account. We will show, using Monte Carlo simulation, that the single-event factorial moments are saturated by the statistical fluctuations when the multiplicity is low. The phenomena observed in experiments [10], which were interpreted as a signal of chaos can readily be reproduced by a flat probability distribution with only statistical fluctuations. The idea [6] of studying chaos in high energy collisions using the distribution width of single-event moments in event space is meaningful only for high multiplicity events. 
In order to study the influence of statistical fluctuations on the chaos-study using singleevent moments, let us rephrase the formalism [6] in terms of both the factorial moments and the probability moments parallelly.

The factorial moment $F_{q}^{(\mathrm{e})}$ and probability moment $C_{q}^{(\mathrm{e})}$ for each event are defined as

$$
\begin{aligned}
F_{q}^{(\mathrm{e})} & =\frac{\frac{1}{M} \sum_{m=1}^{M} n_{m}\left(n_{m}-1\right) \cdots\left(n_{m}-q+1\right)}{\left(\frac{1}{M} \sum_{m=1}^{M} n_{m}\right)^{q}} \\
C_{q}^{(\mathrm{e})} & =M^{q-1} \sum_{i=1}^{M}\left(p_{i}^{(\mathrm{e})}\right)^{q}
\end{aligned}
$$

respectively.

The moments $F_{q}^{(\mathrm{e})}$ (or $C_{q}^{(\mathrm{e})}$ ) may fluctuate greatly from event to event. In a sample consisting of a large number $\mathcal{N}$ of events, we get a distribution $P\left(F_{q}^{(\mathrm{e})}\right)\left(\right.$ or $\left.P\left(C_{q}^{(\mathrm{e})}\right)\right)$, which is normalized to unity. By taking the normalized moments of these distributions in event-space defined as

$$
C_{p, q}^{(\mathrm{F})}=\left\langle F_{q}^{(e)^{p}}\right\rangle /\left\langle F_{q}^{(e)}\right\rangle^{p}, \quad C_{p, q}^{(\mathrm{C})}=\left\langle C_{q}^{(e)^{p}}\right\rangle /\left\langle C_{q}^{(e)}\right\rangle^{p}
$$

we have a quantification of the fluctuation of the spatial patterns, i.e. we can investigate the full shape of the distribution and, especially, the way it changes with the resolution $\delta=\Delta / M$. The value of $p$ in eqn.(3) can take any positive real number. If $C_{p, q}^{(\mathrm{F})}(M)$ (or $\left.C_{p, q}^{(\mathrm{C})}(M)\right)$ has a power law behaviour in $M$, i.e.

$$
C_{p, q}^{(\mathrm{F})}(M) \propto M^{\psi_{q}^{(\mathrm{F})}(p)}, \quad C_{p, q}^{(\mathrm{C})}(M) \propto M^{\psi_{q}^{(\mathrm{C})}(p)}
$$

then the entropy index can be defined as, (For simplicity in notation, the superscript (F) and $(\mathrm{C})$ will be omitted in the following.)

$$
\mu_{q}=\left.\frac{d}{d p} \psi_{q}(p)\right|_{p=1}
$$

It is easy to see that finite, nonvanishing positive values of $\mu_{q}$ corresponds to wide distribution of $F_{q}^{(\mathrm{e})}$ (or $C_{q}^{(\mathrm{e})}$ ), which in turn means unpredictable spatial pattern from event to 
event. By applying the measure to known classical chaotic system, it has been shown [6] that $\mu_{q}$ can be used as a measure of chaos in problems where only the spatial patterns can be observed and the positivity of $\mu_{q}$ is a criterion for chaos.

An alternative way of calculating $\mu_{q}[8]$ is to express $C_{p, q}$ as

$$
C_{p, q}=\left\langle\Phi_{q}^{(e)^{p}}\right\rangle
$$

in which,

$$
\Phi_{q}^{(e)}=C_{q}^{(e)} /\left\langle C_{q}^{(e)}\right\rangle
$$

With the definition

$$
\Sigma_{q}=\left\langle\Phi_{q}^{(e)} \ln \Phi_{q}^{(e)}\right\rangle
$$

we can obtain

$$
\mu_{q}=\frac{\partial \Sigma_{q}}{\partial \ln M}
$$

in the scaling region, i.e. where $\Sigma_{q}$ exhibits a linear dependence on $\ln M$.

In order to study the influence of statistical fluctuations let us turn now to the application of this formalism to a simple model of dynamical fluctuations — the random cascading $\alpha$ model [3] [11] and consider both the case of pure probability distribution without any particle distributed in the space and the case with particle distribution.

In the random cascading $\alpha$-model, the $M$ divisions of a phase space region $\Delta$ are made in steps. At the first step, it is divided into two equal parts; at the second step, each part in the first step is further divided into two equal parts, and so on. The steps are repeated until $M=\Delta Y / \delta y=2^{\nu}$. How particles are distributed from step-to-step between the two parts of a given phase space cell is defined by independent random variable $\omega_{\nu j_{\nu}}$, where $j_{\nu}$ is the position of the sub-cell $\left(1 \leq j_{\nu} \leq 2^{\nu}\right)$ and $\nu$ is the number of steps. It is given by [11]:

$$
\omega_{\nu, 2 j-1}=\frac{1}{2}(1+\alpha r) \quad ; \quad \omega_{\nu, 2 j}=\frac{1}{2}(1-\alpha r), \quad j=1, \ldots, 2^{\nu-1}
$$


where, $r$ is a random number distributed uniformly in the interval $[-1,1] . \alpha$ is a positive number less than unity, which determines the region of the random variable $\omega$ and describes the strength of dynamical fluctuations in the model. After $\nu$ steps, the probability in the $m$ th window $(m=1, \ldots, M)$ is $p_{m}=\omega_{1 j_{1}} \omega_{2 j_{2}} \ldots \omega_{\nu j_{\nu}}$. A certain number $N$ of particles will then be put in the $M$ sub-cells according to the multinomial (Bernouli) distribution

$$
B\left(n_{1}, \ldots, n_{M} \mid p_{1}, \ldots, p_{M}\right)=\frac{N !}{n_{1} ! \cdots n_{M} !} p_{1}^{n_{1}} \cdots p_{M}^{n_{M}}, \quad \sum_{m=1}^{M} n_{m}=N
$$

The factorial moment $F_{q}^{(e)}$ and probability moment $C_{q}^{(e)}$ in each event can now be calculated according to eqn.(1) and (2) respectively. Then the moment $C_{p, q}$ and entropy index $\mu_{q}$ of the sample can be obtaioned using eqn.(3) and eqn.(9).

Firstly, let us consider the case of pure probability distribution without any particle. It can be shown [12] that when the model parameter $\alpha$ is fixed to a constant value there will not be any chaotic behaviour, i.e. the entropy index $\mu$ will vanish. A positive $\mu$ together with the anomalous scaling of averaged moment (intermittency) can be obtained when $\alpha$ is distributed over a certain range. For simplicity let $\alpha$ be a random variable having a Gaussian distribution. The mean and variance of the Gaussian are both chosen as 0.22 . The results of $\ln C_{2}, \ln C_{p, 2}$ and $\Sigma_{2}$ as function of $\ln M$ are shown in Fig.1(a). It can be seen from the figure that both the intermittency (power-law behavior of the averaged moment $C_{2}$ ) and the chaos (diverse of $C_{p, 2}$ for the increasing of $M$ ) are reproduced. The intermittency index $\phi_{2}$ and entropy index $\mu_{2}$ obtained through linear fit of $\ln C_{2}$ and $\Sigma_{2}$ vs. $\ln M$ (in the latter case only the last 3 points are used in the fit) are:

$$
\phi_{2}=0.056, \quad \mu_{2}=0.016
$$

respectively.

Now let us put in a certain number, say 9, of particles according to the Bernouli distribution, cf. eqn.(11). The results of $\ln F_{2}, \ln C_{p, 2}$ and $\Sigma_{2}$ as function of $\ln M$ are shown 
in Fig.1(b). It can be seen from the figure that both the intermittency and the chaos are survived. However, the scale of $C_{p, 2}$ increases for an order of magnitude, showing a much stronger diverse of $C_{p, 2}$ with the increasing of $M$. The intermittency index $\phi_{2}$ and entropy index $\mu_{2}$ obtained in the same way as do in the previous no-particle case are

$$
\phi_{2}=0.058, \quad \mu_{2}=0.41
$$

respectively.

Comparing eqn.(13) with eqn.(12) we see that the anomalous scaling of the averaged factorial moment $F_{2}$ is very similar to that of the averaged probability moment $C_{2}$. The corresponding intermittency indices are almost equal. This means that in the moments averaged over event sample the statistical fluctuations are very well eliminated by the factorial moment method [3]. On the contrary, the entropy index $\mu_{2}$ for the 9-particle case is much bigger than that for the pure probability case, bigger for order of magnitude. This large "entropy index" is clearly comes from statistical fluctuations.

In order to convince us of the dominant role of statistical fluctuations let us consider the case with perfectly flat probabilty distribution, i.e. $p_{m}=1 / M(m=1, \ldots, M)$, without any dynamical fluctuation. In this case, calculating with probability moments, both the averaged $C_{q}$ and the $C_{p, q}$ are identically equal to unity, and the $\ln C_{q}, \ln C_{p, q}$ and $\Sigma_{q}$ all vanish, i.e. no intermittency, no chaos.

After putting in 9 particles, the averaged factorial moments keep constant approximately, independent on $M$, cf. the upper figure of Fig.1(c). (Its value moving down from zero is due to the particular normalization used.) Therefore, the intermittency index almost vanishes as expected. However, the $C_{p, 2}$ diverse strongly, cf. the middle figure of Fig.1(c). The intermittency and entropy indices obtained through linear fit are

$$
\phi_{2}=0.00046, \quad \mu_{2}=0.49
$$


respectively. This large value of $\mu_{2}$ is totally due to statistical fluctuations. Note that in getting this result the random cascading $\alpha$ model has not been used and therefore the conclusion is model-independent.

The results of flat probability distribution with 9 particles are compared with those from the NA27 data [10] in Fig.2. It can be seen from the figure that the fit is very well, which means that the phenomena observed in this experiment, being dominated by statistical fluctuations, does not indicate the existence of chaos.

The particle number 9 used above is about the average multiplicity of the moderateenergy hadron-hadron collision experiments, such as NA27 and NA22. When the number of particle increases, the influence of statistical fluctuations decreases. In Fig.3 are shown the "entropy indices" $\mu_{q}$ of flat probability distribution with different number $N$ of particles. It can be seen that $\mu_{q}$ decreases with the increasing of $N$ and tends to zero when $N$ goes larger and larger. At the upper-right corner of Fig.3 are plotted the fits of $\mu_{q}$ vs. $N$ to the empirical formula

$$
\mu_{q}=A_{q} \mathrm{e}^{-b_{q} N^{0.2}}
$$

The fitting parameters are listed in Table I.

In Fig. 4 are shown the dependence of second order entropy index $\mu_{2}$ on the multiplicity for both the flat probability distribution and the $\alpha$ model with Gaussian distributed $\alpha$. It can be seen from the figure that when multiplicity is low, e.g. lower than 50, the two cases are almost undistinguishable. It is worthwhile noticing that in this case the 'entropy index' $\mu_{2}$ for flat probability distribution is even higher than that for Gaussian distributed $\alpha$. This means that the existence of chaotic behavior cannot be revealed using this method. When multiplicty is high, e.g. higher than 200, the entropy index $\mu_{2}$ for Gaussian distributed $\alpha$ becomes greater than that for flat probability distribution and approaches the physically meaningful entropy index of probability distribution (solid line of Fig.4). 
It is interesting to try this kind of analysis with the central collision data of relativistic heavy ion collision experiments, where the multiplicities are very high. If the entropy indices $\mu_{q}$ obtained in these experiments turn out to be considerably bigger than the value for flat probability distribution, shown in Fig. 3 and the full circles of Fig.4, then that will be a signal of some interesting new physics.

Table I Fitting parameters of $\mu_{q}$ vs. $N$ to eqn.(15)

\begin{tabular}{|c|c|c|}
\hline$q$ & $A_{q}$ & $b_{q}$ \\
\hline 2 & 2.124 & 3.954 \\
\hline 3 & 2.397 & 3.463 \\
\hline 4 & 2.526 & 3.072 \\
\hline
\end{tabular}

In summary, it is shown in this letter using Monte Carlo simulation that the single-event factorial moments are saturated by the statistical fluctuations. The diverse of the event-space moments $C_{p, q}$ of single-event moments with the diminishing of phase space scale (erraticity) observed in experiment can readily be reproduced by a flat probability distribution with only statistical fluctuations and therefore does indicate the existence of chaos as suggested. Using "erraticity analysis" to study chaos in high energy collisions is meaningful only for high multiplicity events.

\section{Acknowledgement}

The authors are grateful to R. Hwa for helpful discussions. 
Appendix On the elimination of statistical fluctuations in the vertically and horizontally averaged factorial moments

Firstly, let us recall the elimination of statistical fluctuations in the vertically averaged FM briefly. The basic assumption was that the statistical fluctuations are Poissonian:

$$
Q_{n}=\int_{0}^{\infty} \frac{s^{n}}{n !} \mathrm{e}^{-s} D(s) \mathrm{d} s
$$

where $Q_{n}$ is the multiplicity distribution in the $i$ th bin, $D(s)$ the dynamical distribution in the same bin. In eqn.(A1) we have used the notation $n$ with no subscript to denote the number of particles in the $i$ th bin [8] The multiplicity distribution $Q_{n}$ and the dynamical distribution $D(s)$ are restricted by the normalization conditions

$$
\begin{gathered}
\sum_{n=0}^{\infty} Q_{n}=1, \\
\int_{0}^{\infty} D(s) \mathrm{d} s=1 .
\end{gathered}
$$

In order to check whether the equation (A1) is consistent with the conditions (A2) and (A3), insert (A1) into (A2)

$$
\sum_{n=0}^{\infty} Q_{n}=\int_{0}^{\infty}\left(\sum_{n=0}^{\infty} \frac{s^{n}}{n !} \mathrm{e}^{-s}\right) D(s) \mathrm{d} s .
$$

Using the normalization of Poisson distribution

$$
\sum_{n=0}^{\infty} \frac{s^{n}}{n !} \mathrm{e}^{-s}=1
$$

we get

$$
\sum_{n=0}^{\infty} Q_{n}=\int_{0}^{\infty} D(s) \mathrm{d} s
$$

consistent with eqn.(A2) and (A3).

It should be noticed, however, that the normalization (A5) of Poisson distribution is valid when and only when the summation for $n$ is over all the positive integer (and 0 ) values 
of $n$. If some terms in the summation are missing, or if the summation is ended at some value $n_{\max }$ of $n$, then it will be no longer equal to unity.

Let us consider the elimination of statistical fluctuations in the 2nd order FM as example. Consider only the numerator $f_{2} \equiv n^{[2]} \equiv n(n-1)$ in the definition eqn.(1), and neglect the denorminator, which is only for normalization. We have

$$
\left\langle f_{2}\right\rangle_{\mathrm{v}} \equiv\left\langle n^{[2]}\right\rangle_{\mathrm{v}}=\frac{1}{\mathcal{N}} \sum_{e=1}^{\mathcal{N}} n_{i}^{(e)}\left(n_{i}^{(e)}-1\right)
$$

The crucial point is that, when the number of event $\mathcal{N}$ in a sample is big, the bin-multiplicity $n_{i}^{(e)}(\equiv n)$ for fixed $i$ can take value from zero to very large (constrainted only by energy conservation). Therefore, we have

$$
\left\langle f_{2}\right\rangle_{\mathrm{v}}=\sum_{n=0}^{\infty} n(n-1) Q_{n}=\sum_{n=0}^{\infty} \int_{0}^{\infty} n(n-1) \frac{s^{n}}{n !} \mathrm{e}^{-s} D(s) \mathrm{d} s=\int_{0}^{\infty} \sum_{n=0}^{\infty} \frac{s^{n}}{(n-2) !} \mathrm{e}^{-s} D(s) \mathrm{d} s .
$$

Let $n-2=n^{\prime}$ and make use of the normalization (A5) of Poisson distribution we get

$$
f_{2} \equiv\left\langle f_{2}\right\rangle_{\mathrm{v}}=\int_{0}^{\infty}\left(\sum_{n^{\prime}=0}^{\infty} \frac{s^{n^{\prime}}}{n^{\prime} !} \mathrm{e}^{-s}\right) s^{2} D(s) \mathrm{d} s=\int_{0}^{\infty} s^{2} D(s) \mathrm{d} s \equiv c_{2} .
$$

This is the well known result —_ (vertically averaged) factorial moment (FM) equal to dynamical moment (DM), i.e. the statistical fluctuations have been eliminated successfully in vertically averaging over event sample.

Let us check whether this conclusion is applicable also to horizontally averaging. There are 2 points that are different to the previous vertically averaging case.

1) The number $M$ of bins, unlike the number $\mathcal{N}$ of events, cannot be very large, may be only $1,2, \ldots$, or at most some tens or hudreds.

2) Even more important, the total multiplicity $N^{(e)}$ in a single event is always fixed.

We can of course define a bin-multiplicity distribution $Q_{n}^{(e)}$ of a single event as the distribution of bin-multiplicity $n\left(\equiv n_{i}^{(e)}\right)$ for fixed $e$ in the $M$ bins, but $Q_{n}^{(e)}$ is nonzero only for at most $M^{\prime}$ different values of $n$, where $M^{\prime} \leq M$.

$$
Q_{n}^{(e)} \neq 0 \text { only for } n=n_{1}, n_{2}, \ldots, n_{M^{\prime}} \text {. }
$$


Among the $M^{\prime}$ integers $n_{1}, n_{2}, \ldots, n_{M^{\prime}}$, there is certainly a maximum one $n_{\max }$.

$$
Q_{n}^{(e)}=0 \text { when } n>n_{\max }
$$

Therefore, for the single event bin-multiplicity distribution,

$$
\sum_{n=0}^{\infty} Q_{n}^{(e)}=\int_{0}^{\infty}\left(\sum_{n=0}^{n_{\max }} \frac{s^{n}}{n !} \mathrm{e}^{-s}\right) D(s) \mathrm{d} s
$$

Since the summation over $n$ in the round bracket ends at a finite number $n_{\max }$ and has only $M^{\prime}$ terms, it does not equal to unity and

$$
\sum_{n=0}^{\infty} Q_{n}^{(e)} \neq \int_{0}^{\infty} D(s) \mathrm{d} s
$$

This means that eqn.(A1) is inconsistent with eqn.(A2) and (A3) for $Q_{n}^{(e)}$. In other words, for the single-event bin-multiplicity distribution $Q_{n}^{(e)}$ normalized as eqn.(A2) no normalizable $D(s)$ which satisfies eqn.(A1) can exist. Therefore, the proof for the elimination of statistical fluctuations is no longer valid in the horizontal averaging case.

State alternatively, if we write

$$
\left\langle f_{2}\right\rangle_{\mathrm{h}}=\sum_{n=1}^{\infty} Q_{n}^{(e)} n(n-1)
$$

then due to eqn.(A9) the summation in $n$ doesnot really extend to infinity. If we neglect the non-existence of $D(s)$ for a while and try to do the calculation similar to eqn.(A7),(A8), we will get

$$
\begin{aligned}
\left\langle f_{2}\right\rangle_{\mathrm{h}} & =\sum_{n=1}^{n_{\max }} n(n-1) Q_{n}^{(e)}=\sum_{n=1}^{n_{\max }} \int_{0}^{\infty} n(n-1) \frac{s^{n}}{n !} \mathrm{e}^{-s} D(s) \mathrm{d} s \\
& =\int_{0}^{\infty}\left(\sum_{n=1}^{n_{\max }} \frac{s^{n}}{(n-2) !} \mathrm{e}^{-s}\right) D(s) \mathrm{d} s=\int_{0}^{\infty}\left(\sum_{n^{\prime}=1}^{n_{\max }-2} \frac{s^{n^{\prime}}}{n^{\prime} !} \mathrm{e}^{-s}\right) s^{2} D(s) \mathrm{d} s .
\end{aligned}
$$

The normalization condition (A5) cannot be used here, and therefore

$$
\left\langle f_{2}\right\rangle_{\mathrm{h}} \neq \int_{0}^{\infty} s^{2} D(s) \mathrm{d} s
$$




\section{References}

[1] T. H. Burnett et al., Phys. Rev. Lett. 50 (1983) 2062.

[2] E.A. De Wolf, I.M. Dremin and W. Kittel, Phys. Rep. 270 (1996) 1.

[3] A. Białas and R. Peschanski, Nucl. Phys. B 273 (1986) 703; ibid 308 (1988) 857;

[4] N.M. Agababyan et al. (NA22), Phys. Lett. B382 (1996) 305; ibid B431 (1998) 451;

[5] S. Wang, Z. Wang and C. Wu, Phys. Lett. B 410 (323) 1997.

[6] Z. Cao and R. Hwa, Phys. Rev. Lett. 75 (1995) 1268.

[7] Z. Cao and R. Hwa, Phys. Rev. D 54 (1996) 6674.

[8] Z. Cao and R. Hwa, Phys. Rev. E 56 (1997) 326.

[9] Z. Cao and R. Hwa, Erraticity analysis of soft production by ECOMB, University of Oregon preprint OITS 666, 1999.

[10] Wang Shaushun and Wang Zhaomin, Phys. Rev. D 57 (1998) 3036.

[11] Wu Yuanfang, Zhang Kunshi and Liu Lianshou, Chinese Science Bulletin 36 (1991) 1077.

[12] Fu Jinghua, Wu Yuanfang and Liu Lianshou, On the intermittency and chaos in high energy collisions, to be published. 


\section{Figure Captions}

Fig.1 Logarithm of averaged moment $\ln C_{2}\left(\ln F_{2}\right), \ln C_{p, 2}$ and $\Sigma_{2}$ as function of $\ln M$ for $(a)$ the probability moments of $\alpha$ model with Gaussian-distributed $\alpha ;(b)$ the factorial moments of the same model with 9 particles put in; $(c)$ the factorial moments of a fat probability distribution with 9 particles put in. solid lines are linear fit. Dashed lines are for guiding the eye.

Fig.2 $\ln C_{p, q}(q=2-4)$ vs. $\ln M$ for a flat distribution with 9 particles put in (full squares) compared with the experimental results of NA27 data (open circles). Data taken from Ref.[10].

Fig.3 The dependence of the entropy indices $\mu_{q}$ on the number of particles. The solid lines are the results from the empirical formula (15) and Table I.

Fig.4 The dependence on the number of particles of the entropy indices $\mu_{2}$ calculated from factorial moments. Full circles — flat probability distribution with only statistical fluctuations; open triangles — Gaussian distributed $\alpha$. The solid line is the result from probability moments with Gaussian distributed $\alpha$. The dashed lines are for guiding the eye. 

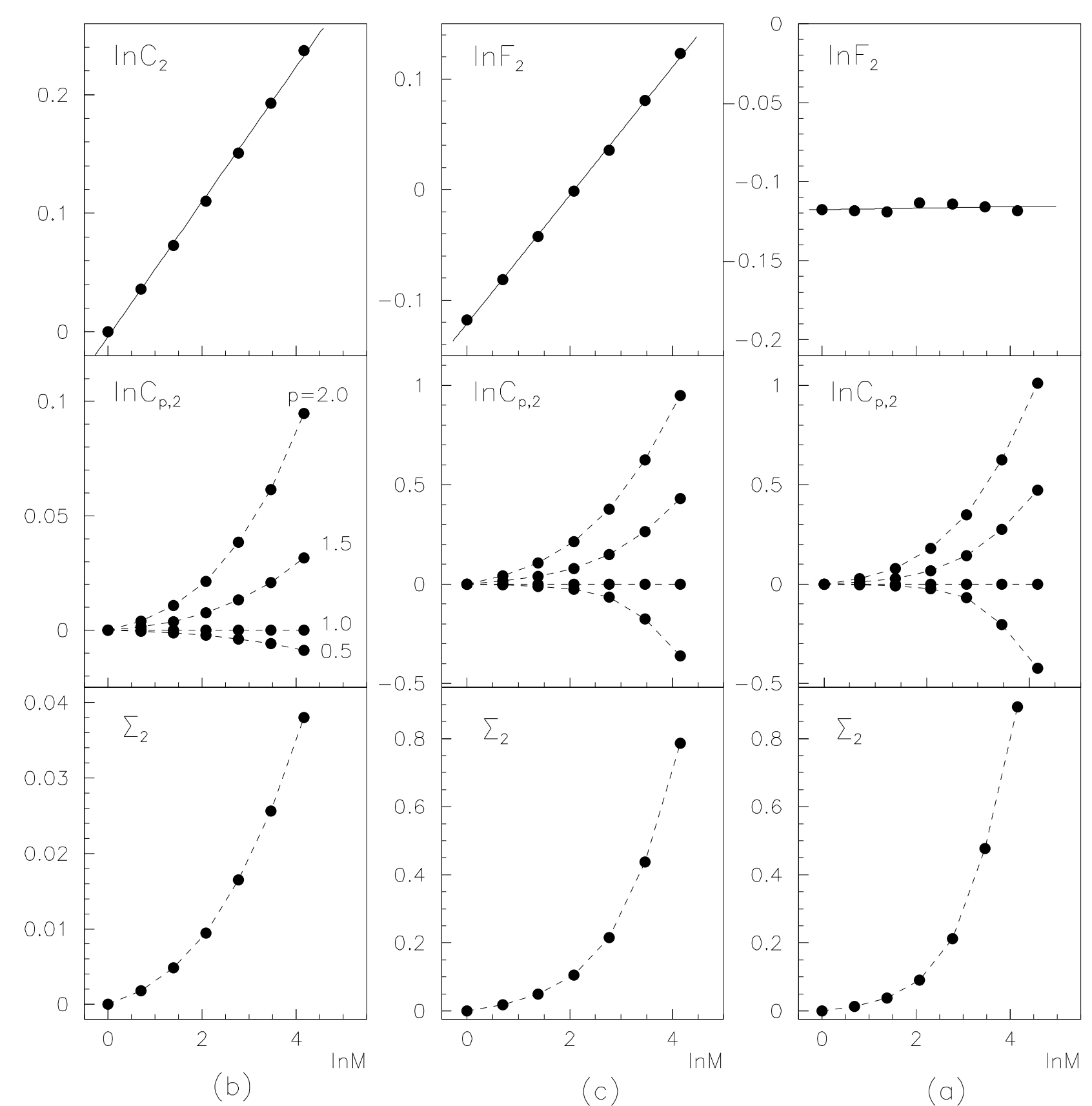

Fig.1 Logarithm of averaged moment $\ln C_{2}\left(\ln F_{2}\right)$ and $\ln C_{p, 2}$ as function of $\ln M$ for $(a)$ the probability moments of $\alpha$ model with Gaussian-distributed $\alpha$; (b) the factorial moments of the same model with 9 particles put in; $(c)$ the factorial moments of a fat probability distribution with 9 particles put in. Solid lines are linear fit. Dashed lines are for guiding the eye. 

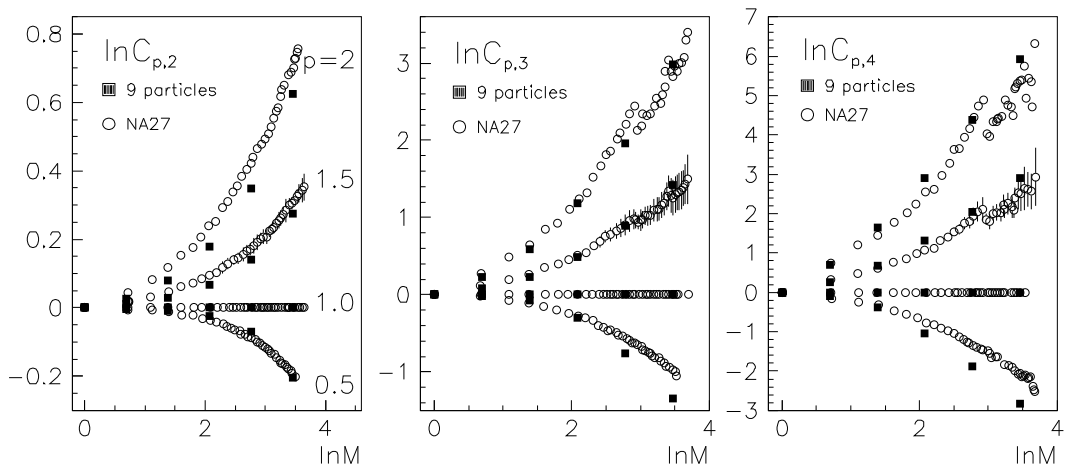

Fig. $2 \ln C_{p, q}(q=2-4)$ vs. $\ln M$ for a flat distribution with 9 particles put in (full squares) compared with the experimental results of NA27 data (open circles). Data taken from Ref. [10].

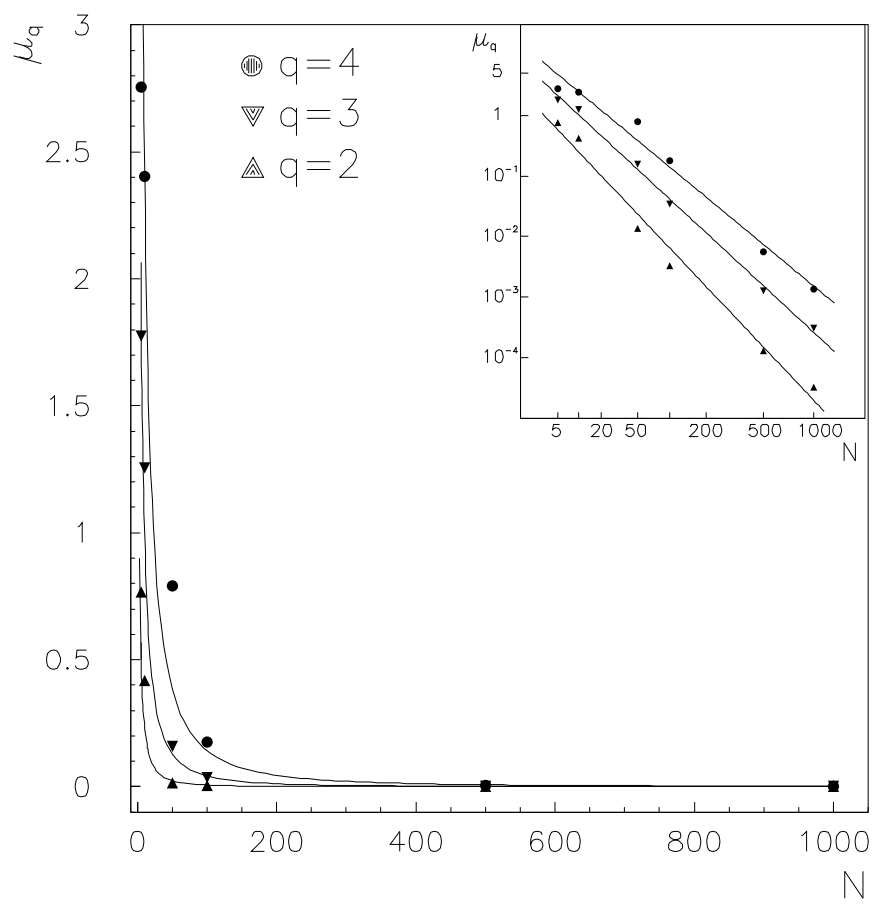

Fig.3 The dependence of the entropy indices $\mu_{q}$ on the number of particles. The solid lines are the results from the empirical formula (15) and Table I. 


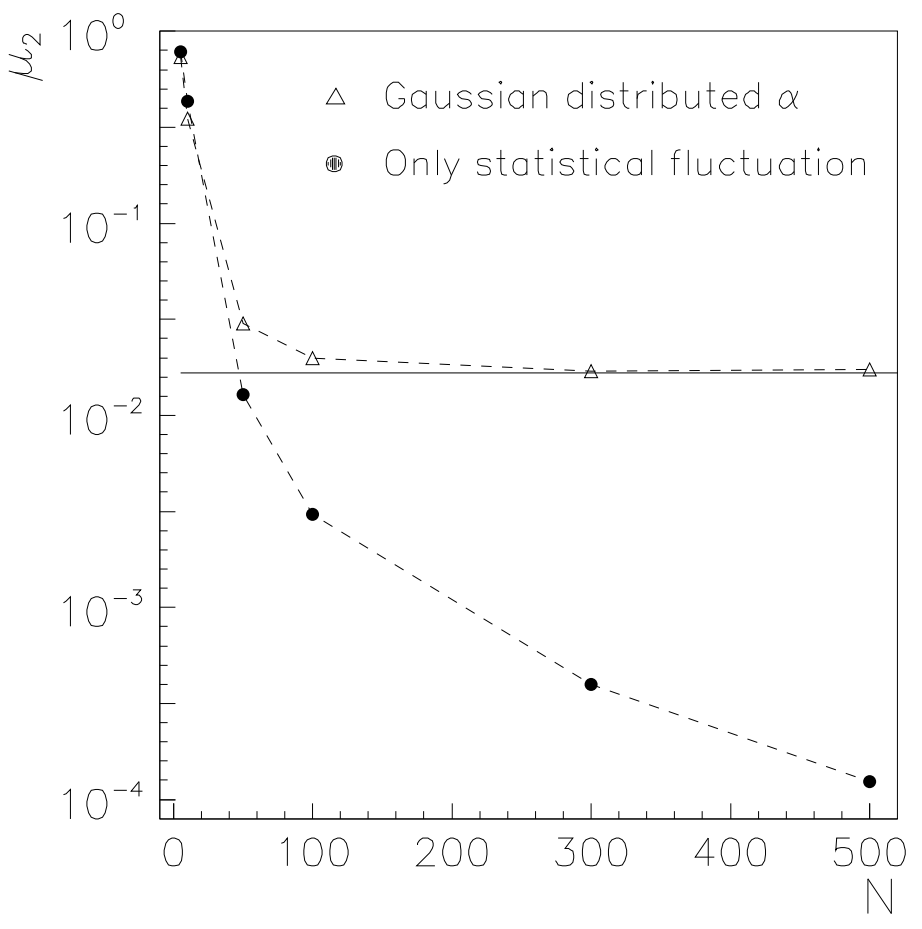

Fig.4 The dependence on the number of particles of the entropy indices $\mu_{2}$ calculated from factorial moments. Full circles — flat probability distribution with only statistical fluctuations; open triangles Gaussian distributed $\alpha$. The solid line is the result from probability moments with Gaussian distributed $\alpha$. The dashed lines are for guiding the eye. 